\title{
A transmutation operator method for solving the inverse quantum scattering problem
}

\author{
Vladislav V. Kravchenko ${ }^{1}$, Elina L. Shishkina ${ }^{2}$ and Sergii M. Torba ${ }^{1}$ \\ ${ }^{1}$ Departamento de Matemáticas, Cinvestav, Unidad Querétaro, \\ Libramiento Norponiente \#2000, Fracc. Real de Juriquilla, Querétaro, Qro., 76230 MEXICO. \\ ${ }^{2}$ Voronezh State University. \\ e-mail: vkravchenko@math.cinvestav.edu.mx, storba@math.cinvestav.edu.mx, *
}

September 25, 2020

\begin{abstract}
The inverse quantum scattering problem for the perturbed Bessel equation is considered. A direct and practical method for solving the problem is proposed. It allows one to reduce the inverse problem to a system of linear algebraic equations, and the potential is recovered from the first component of the solution vector of the system. The approach is based on a special form Fourier-Jacobi series representation for the transmutation operator kernel and the Gelfand-Levitan equation which serves for obtaining the system of linear algebraic equations. The convergence and stability of the method are proved as well as the existence and uniqueness of the solution of the truncated system. Numerical realization of the method is discussed. Results of numerical tests are provided revealing a remarkable accuracy and stability of the method.
\end{abstract}

\section{Introduction}

We present a direct and simple method for practical solution of the inverse quantum scattering problem for the perturbed Bessel equation

$$
L u:=-u^{\prime \prime}+\left(\frac{\ell(\ell+1)}{x^{2}}+q(x)\right) u=\rho^{2} u, \quad x>0
$$

with an arbitrary fixed angular momentum $\ell \geq-1 / 2$ and the potential $q$ satisfying

$$
\int_{0}^{\infty}\left(x^{\mu}+x\right)|\tilde{q}(x)| d x<+\infty
$$

for some $\mu \in[0,1 / 2)$, where

$$
\tilde{q}(x)= \begin{cases}q(x), & \ell>-1 / 2, \\ (1+|\log (x)|) q(x), & \ell=-1 / 2 .\end{cases}
$$

The problem consists in recovering $q$ from the given scattering data. The bibliography dedicated to the theory of this problem and applications is vast. We refer to [2], [5], [7], [26], [18] and references

\footnotetext{
${ }^{*}$ Research was supported by CONACYT, Mexico via the projects 222478 and 284470 . Research of Vladislav Kravchenko was supported by the Regional mathematical center of the Southern Federal University, Russia.
} 
therein. However, the numerical solution of the problem presents difficulties. We refer the reader to [3] and [25] where numerical approaches are discussed, although the way of presenting the numerical results does not give us a possibility to make a comparison.

The method presented in this paper allows one a direct reduction of the inverse quantum scattering problem to a system of linear algebraic equations. Moreover, only the first component of the solution vector is necessary to recover the potential. The method is simple and does not require much programmer's work. It is based on the classical results from the spectral theory, such as the Gelfand-Levitan equation and the transmutation operator, as well as on a new functional series representation for the transmutation integral kernel, obtained in [24].

The present work extends the applicability of the approach based on the functional series representations for the transmutation integral kernels developed in the regular case $\ell=0$ in [20], 21, 8], [15] and reported in the book [22]. The extension of an approach onto the singular case $\ell \neq 0$ is always a challenge requiring additional ideas and tools. The first important ingredient here is an appropriate Fourier-Jacobi series representation for the transmutation operator kernel [24]. It captures singular features of the kernel, such as its behaviour near $t=0$ and on the characteristic line $t=x$, and allows one to recover the potential from the first coefficient of the series. Thus, we do not follow the usual approach of computing the transmutation kernel first and then recovering the potential from it. Instead, we compute the first coefficient of the Fourier-Jacobi series representation, from which the potential is recovered.

The right choice of the orthogonal function system used in the series representation resulted to be of crucial importance in the interplay between the transmutation operator kernel and the GelfandLevitan input kernel, which gave us the possibility in the present work to obtain a system of linear algebraic equations for the coefficients of the series representation with explicit formulas for the entries of the system matrix.

We prove the convergence and stability of the method. This results in the possibility of recovering the potential from noisy scattering data. A corresponding numerical example is provided. Moreover, we prove the existence and uniqueness of the solution of the truncated system of equations arising in the numerical realization of the method.

Thus, the method developed in the present work is convergent, stable and possesses an important additional advantage. Its numerical implementation is simple and does not require much programmer effort. The numerical examples reveal a remarkable accuracy, stability and fast convergence of the method.

Besides this introduction the paper contains four sections. Section 2 presents some preliminaries on the inverse quantum scattering problem including the example of the square well potential, which is used later on for one of the numerical tests. In Section 3 the Fourier-Jacobi series representation for the transmutation operator kernel is presented. It is explained how the potential can be recovered from the first coefficient of the series, and the Gelfand-Levitan equation is recalled. In Section 4 we construct the system of linear algebraic equations for the coefficients of the Fourier-Jacobi series representation, prove the existence and uniqueness of solutions of corresponding truncated systems and the convergence of solutions of truncated systems to the exact one. Observing that the obtained truncated systems result from applying the Bubnov-Galerkin procedure with a special choice of the orthogonal function system, we prove the stability of the method, which allows one to work efficiently with noisy scattering data. In Section 5 we discuss the numerical implementation of the method and provide some numerical examples. They illustrate that indeed the developed approach leads to a direct and simple method for accurate recovering of the potential even with few equations in the truncated system and from noisy scattering data. Finally, in Appendix A we present a refined asymptotics of the Jost function. 


\section{Preliminaries}

We consider the perturbed Bessel equation

$$
L u:=-u^{\prime \prime}+\left(\frac{\ell(\ell+1)}{x^{2}}+q(x)\right) u=\rho^{2} u, \quad x>0
$$

with the coefficient $q$, often called the potential, being a real valued function satisfying the condition

$$
\left(x^{\mu}+x\right) \tilde{q}(x) \in L_{1}(0, \infty) \quad \text { for some } 0 \leq \mu<1 / 2,
$$

Here $\tilde{q}$ is given by (1.2). Sometimes, potentials satisfying (2.2) at infinity are said to belong to the Marchenko class. The spectral parameter $\rho \in \mathbb{C}$ is chosen so that $\operatorname{Im} \rho \geq 0$ and $\ell \geq-1 / 2$.

We are interested in a procedure for solving the inverse quantum scattering problem consisting in recovering a potential $q(x)$ in the perturbed Bessel equation from so-called scattering data which include the eigenvalues, the corresponding norming constants and the Jost function $F_{\ell}(\rho), \rho \in[0, \infty)$. Notice that we suppose the Jost function to be given, although in a usual study of the inverse problem it is obtained first from the $S$-function (the scattering function) which is supposed in its turn to be known as a part of the scattering data.

The unique solvability of such inverse quantum scattering problem follows from [18, Theorem 5.1], where a more general class of potentials is considered for arbitrary $\ell \geq-1 / 2$. Additional restrictions on the potential imposed in this paper are needed to guarantee that the problem possesses at most a finite number of eigenvalues, to use the Gelfand-Levitan equation and to be sure that the solution of the Gelfand-Levitan equation is square-integrable. For the case of integer $\ell$ one can consult a lot of additional details, e.g., in [36] and [5].

We remind that the set of eigenvalues, if it is not empty, consists of a finite set of numbers $\rho_{j}^{2} \leq 0$, $j=1, \ldots, N$, which are such that equation (2.1) admits a square integrable solution on $(0, \infty)$, see [5, (II.1.10a)], [32, Theorem 5.1] and [37, Section 9.7]. Thus, $\rho_{j}=i \tau_{j}, \tau_{j} \geq 0$. For recalling the definition of the norming constants and of the Jost function we proceed with some necessary notations.

A solution $\varphi_{\ell}(\rho, x)$ of (2.1) satisfying the asymptotic relation at the origin

$$
\lim _{x \rightarrow 0} \frac{2^{\ell+1}}{\sqrt{\pi}} \Gamma\left(\ell+\frac{3}{2}\right) x^{-(\ell+1)} \varphi_{\ell}(\rho, x)=1,
$$

is called the regular solution. Note that for integer values of $\ell$ one has $\frac{2^{\ell+1}}{\sqrt{\pi}} \Gamma\left(\ell+\frac{3}{2}\right)=(2 \ell+1) !$ !. The last formula is known as the extension of the double factorial symbol to complex arguments. To simplify notations, later in this paper we will use $(2 \ell+1) ! !$.

In the case when $\rho=\rho_{j}$ is an eigenvalue, the regular solution $\varphi_{\ell}\left(\rho_{j}, x\right)$ is an eigenfunction, and the norming constants are defined as

$$
c_{j}:=\frac{1}{\int_{0}^{\infty} \varphi_{\ell}^{2}\left(\rho_{j}, x\right) d x} .
$$

A solution $f_{\ell}(\rho, x)$ of (2.1) satisfying the asymptotic relation at infinity

$$
\lim _{x \rightarrow \infty}\left(e^{-\frac{i \pi \ell}{2}} e^{-i \rho x} f_{\ell}(\rho, x)\right)=1
$$

is called the Jost solution. The uniqueness and the existence of both regular and Jost solutions is a well known fact (see, e.g., [5], and for non-integer values of $\ell$, 19], 13] and references therein).

The function $F_{\ell}(\rho)$ which can be represented as a Wronskian of the solutions

$$
F_{\ell}(\rho)=(-\rho)^{\ell} W\left[f_{\ell}(\rho, x), \varphi_{\ell}(\rho, x)\right]
$$


is known as the Jost function. In fact, the Jost function contains information on the behaviour of the Jost solution at the origin. The following asymptotic relation is valid for $\ell>-1 / 2$ (see, e.g., [5, Section 1.5], for non-integer values of $\ell$ it can be established using the results from [19])

$$
F_{\ell}(\rho)=\lim _{x \rightarrow 0} \frac{(-\rho x)^{\ell}}{(2 \ell-1) ! !} f_{\ell}(\rho, x)
$$

while for $\ell=-1 / 2$ it can be deduced (see [13, Subsection 2.1]) that

$$
F_{-1 / 2}(\rho)=\lim _{x \rightarrow 0}-\frac{\sqrt{\pi}(-\rho)^{-1 / 2}}{\sqrt{2 x} \log x} f_{-1 / 2}(\rho, x) .
$$

Note that $F_{\ell}$ is analytic in the upper half-plane, $F_{\ell}(\rho)=1+o(1)$ when $\rho \rightarrow \infty, \operatorname{Im} \rho \geq 0$, and $F_{\ell}(-\rho)=\overline{F_{\ell}}(\rho)$ for $\rho \in \mathbb{R}[18$, Lemma B.5]. Moreover, for $\ell>-1 / 2$ and potentials $q$ such that $q \in L_{1}(0, \infty)$ the asymptotic formula is valid

$$
F_{\ell}(\rho)=1+\frac{i}{2 \rho} \int_{0}^{\infty} q(x) d x+o\left(\rho^{-1}\right), \quad|\rho| \rightarrow \infty
$$

see [19, Remark 2.14]. In Appendix $\mathrm{A}$ we prove a refinement of this formula, namely, that

$$
F_{\ell}(\rho)=1+\frac{i}{2 \rho} \int_{0}^{\infty} q(x) d x+O\left(\rho^{-2}\right), \quad|\rho| \rightarrow \infty
$$

for any $\ell \geq-1 / 2$ and potentials $q \in L_{1}(0, \infty) \cap B V_{0}[0, \infty)$. Here $B V_{0}$ denotes functions of bounded variation vanishing at infinity.

Denote by $b_{\ell}(\rho x)$ a solution of the Bessel equation

$$
-u^{\prime \prime}+\frac{\ell(\ell+1)}{x^{2}} u=\rho^{2} u, \quad x>0
$$

satisfying the asymptotic relation

$$
b_{\ell}(\rho x) \sim \frac{(\rho x)^{\ell+1}}{(2 \ell+1) ! !}, \quad x \rightarrow 0 .
$$

It has the form

$$
b_{\ell}(\rho x)=\rho x j_{\ell}(\rho x)
$$

where $j_{\ell}$ stands for the spherical Bessel function of the first kind (see [1, Section 10.1]), $j_{\ell}(z):=$ $\sqrt{\frac{\pi}{2 z}} J_{\ell+\frac{1}{2}}(z)$.

Example 2.1. Consider the square well potential $q$ of the form

$$
q(x)= \begin{cases}-Q^{2}, & x \leq R \\ 0, & x>R\end{cases}
$$

where $Q$ is a positive constant. Denote $\omega:=\sqrt{\rho^{2}+Q^{2}}$. Then the Jost solution has the form

$$
f_{\ell}(\rho, x)= \begin{cases}a(\rho) b_{\ell}(\omega x)+b(\rho) \omega x h_{\ell}^{(1)}(\omega x), & x \leq R \\ (-1)^{\ell} i \rho x h_{\ell}^{(1)}(\rho x), & x>R\end{cases}
$$


where the coefficients $a(\rho)$ and $b(\rho)$ are found from the condition of continuity of the solution $f_{\ell}(\rho, x)$ and of its derivative at $x=R$, which leads to the following system of equations

$$
\begin{aligned}
a(\rho) b_{\ell}(\omega R)+b(\rho) \omega R h_{\ell}^{(1)}(\omega R) & =(-1)^{\ell} i \rho R h_{\ell}^{(1)}(\rho R), \\
a(\rho)\left(\omega(\ell+1) j_{\ell}(\omega R)-\omega^{2} R j_{\ell+1}(\omega R)\right) & +b(\rho)\left(\omega(\ell+1) h_{\ell}^{(1)}(\omega R)-\omega^{2} R h_{\ell+1}^{(1)}(\omega R)\right) \\
& =(-1)^{\ell} i \rho\left((\ell+1) h_{\ell}^{(1)}(\rho R)-\rho R h_{\ell+1}^{(1)}(\rho R)\right) .
\end{aligned}
$$

From (2.3) we find that

$$
F_{\ell}(\rho)=(-1)^{\ell+1} i b(\rho)\left(\frac{\rho}{\omega}\right)^{\ell}
$$

\section{The transmutation integral kernel}

A solution $u_{\ell}(\rho, x)$ of (2.1), satisfying the asymptotic relation (2.6) admits the following representation

$$
u_{\ell}(\rho, x)=T\left[b_{\ell}(\rho x)\right]:=b_{\ell}(\rho x)+\int_{0}^{x} K_{\ell}(x, t) b_{\ell}(\rho t) d t
$$

where the integral kernel $K_{\ell}(x, t)$ is a square integrable function of the variable $t$, independent of $\rho$. This Volterra integral operator of the second kind is known as a transmutation (or transformation) operator. The existence of such $K_{\ell}(x, t)$ for the potentials satisfying condition (1.1) at zero was proved in [36]. Properties of $K_{\ell}(x, t)$ were studied in several publications (see, e.g., [38], [7], 6], 12], [16], [23], [33], [34]). For the purpose of the present work the following statement is crucial.

Theorem 3.1 ([24]). Let $q$ satisfy the condition $\int_{0}^{b} x^{\mu}|q(x)| d x<\infty$ for some $0 \leq \mu<1 / 2$. Then the kernel $K_{\ell}(x, t)$ admits the following series representation

$$
K_{\ell}(x, t)=\sum_{n=0}^{\infty} \frac{\beta_{n}(x)}{x^{\ell+2}} t^{\ell+1} P_{n}^{(\ell+1 / 2,0)}\left(1-\frac{2 t^{2}}{x^{2}}\right),
$$

where $P_{n}^{(\alpha, \beta)}$ stands for the Jacobi polynomial and the coefficients $\beta_{n}(x)$ can be calculated by a recurrent integration procedure, starting with

$$
\beta_{0}(x)=(2 \ell+3)\left(\frac{u_{\ell, 0}(x)}{x^{\ell+1}}-1\right)
$$

where $u_{\ell, 0}(x)$ is a regular solution of the equation

$$
L u=0
$$

normalized by the asymptotic condition $u_{\ell, 0}(x) \sim x^{\ell+1}, x \rightarrow 0$.

For any $x>0$, the series in (3.1) converges in $L_{2}(0, x)$. Suppose additionally that $q$ is absolutely continuous on $[0, b]$. Then the series in (3.1) converges absolutely and uniformly with respect to $t \in[0, x-\varepsilon]$ for an arbitrarily small $\varepsilon>0$. If additionally $q \in W_{1}^{3}[0, b]$, then the series converges absolutely and uniformly with respect to $t$ on the whole $[0, x]$.

Remark 3.2. The condition on the potential $q$ in the theorem is equivalent to condition (1.1) at the origin. The recurrent integration procedure mentioned in the theorem is superfluous for the present work and can be consulted in [24]. 
Remark 3.3. Equality (3.2) gives us the possibility to recover the potential $q$ if $\beta_{0}$ is known. Indeed, we have that

$$
u_{\ell, 0}(x)=\left(\frac{\beta_{0}(x)}{(2 \ell+3)}+1\right) x^{\ell+1},
$$

and since $u_{\ell, 0}$ is a solution of (․3.3), we obtain

$$
q=\frac{x \beta_{0}^{\prime \prime}(x)+2(\ell+1) \beta_{0}^{\prime}(x)}{x\left(\beta_{0}(x)+2 \ell+3\right)} .
$$

Remark 3.4. The following orthogonality property of the Jacobi polynomials is valid [24]

$$
\int_{0}^{x} t^{2 \ell+2} P_{n}^{(\ell+1 / 2,0)}\left(1-\frac{2 t^{2}}{x^{2}}\right) P_{m}^{(\ell+1 / 2,0)}\left(1-\frac{2 t^{2}}{x^{2}}\right) d t=\frac{x^{2 \ell+3}}{4 m+2 \ell+3} \delta_{n m}
$$

with $\delta_{n m}$ standing for the Kronecker delta. Consequently, for any $x>0$ fixed, the system of functions

$$
p_{n}(x ; t):=\frac{\sqrt{4 n+2 \ell+3}}{x^{\ell+3 / 2}} t^{\ell+1} P_{n}^{(\ell+1 / 2,0)}\left(1-\frac{2 t^{2}}{x^{2}}\right)
$$

is a complete orthonormal system in $L_{2}(0, x)$. Hence the series (3.1) is an expansion of the kernel $K_{\ell}(x, t)$ with respect to the basis of $L_{2}(0, x)$ represented by the system of functions $\left\{p_{n}(x ; t)\right\}_{n=0}^{\infty}$,

$$
K_{\ell}(x, t)=\sum_{n=0}^{\infty} \alpha_{n}(x) p_{n}(x ; t)
$$

with

$$
\alpha_{n}(x)=\frac{\beta_{n}(x)}{\sqrt{4 n+2 \ell+3} \sqrt{x}} .
$$

In the following we assume that zero is not an eigenvalue of the problem. Then the transmutation kernel $K_{\ell}(x, t)$ is related to the scattering data via the Gelfand-Levitan integral equation

$$
K_{\ell}(x, y)+\Omega_{\ell}(x, y)+\int_{0}^{x} K_{\ell}(x, t) \Omega_{\ell}(t, y) d t=0, \quad x>y
$$

where the input kernel $\Omega_{\ell}(x, y)$ has the form

$$
\begin{gathered}
\Omega_{\ell}(x, y)=\sum_{j=1}^{N} C_{j} b_{\ell}\left(i \tau_{j} x\right) b_{\ell}\left(i \tau_{j} y\right)+\frac{2}{\pi} \int_{0}^{\infty} b_{\ell}(\rho x) b_{\ell}(\rho y)\left(\left|F_{\ell}(\rho)\right|^{-2}-1\right) d \rho, \\
C_{j}:=\frac{c_{j}}{\left(i \tau_{j}\right)^{2 \ell+2}} .
\end{gathered}
$$

Note that under condition (1.1) the integral kernel $K_{\ell}$ satisfies [36] for any finite $a>0$

$$
\sup _{0 \leq x \leq a}\left\|K_{\ell}(x, \cdot)\right\|_{L_{2}(0, x)}^{2}<\infty .
$$

The function $\Omega_{\ell}$ is symmetric, and it can be easily obtained from (3.9) that $\Omega_{\ell}(x, \cdot) \in L_{2}(0, x)$ and $\Omega_{\ell} \in L_{2}((0, x) \times(0, x))$. 


\section{A system of linear algebraic equations for the coefficients $\beta_{n}(x)$}

Denote

$$
A_{m, n}(x):=\sum_{j=1}^{N} C_{j} j_{\ell+2 n+1}\left(i \tau_{j} x\right) j_{\ell+2 m+1}\left(i \tau_{j} x\right)+\frac{2}{\pi} \int_{0}^{\infty} j_{\ell+2 n+1}(\rho x) j_{\ell+2 m+1}(\rho x)\left(\left|F_{\ell}(\rho)\right|^{-2}-1\right) d \rho
$$

and

$$
B_{m}(x):=-\sum_{j=1}^{N} C_{j} b_{\ell}\left(i \tau_{j} x\right) j_{\ell+2 m+1}\left(i \tau_{j} x\right)-\frac{2}{\pi} \int_{0}^{\infty} b_{\ell}(\rho x) j_{\ell+2 m+1}(\rho x)\left(\left|F_{\ell}(\rho)\right|^{-2}-1\right) d \rho .
$$

Theorem 4.1. The coefficients $\beta_{n}$ from (3.1) satisfy the following infinite system of linear algebraic equations

$$
\frac{\beta_{m}(x)}{(4 m+2 \ell+3) x}+\sum_{n=0}^{\infty} \beta_{n}(x) A_{m, n}(x)=B_{m}(x), \quad \text { for all } m=0,1, \ldots
$$

Proof. Let us substitute the representation (3.1) into (3.9). Consider

$$
\int_{0}^{x} K_{\ell}(x, t) \Omega_{\ell}(t, y) d t=\frac{1}{x^{\ell+2}} \sum_{n=0}^{\infty} \beta_{n}(x) \int_{0}^{x} t^{\ell+1} P_{n}^{(\ell+1 / 2,0)}\left(1-\frac{2 t^{2}}{x^{2}}\right) \Omega_{\ell}(t, y) d t .
$$

The possibility of changing the order of summation and integration follows from the observation that this equality is nothing but a concrete realization of the general Parseval identity [4, p. 16]. Indeed, with the aid of Remark 3.4 we have

$$
\begin{aligned}
\int_{0}^{x} K_{\ell}(x, t) \Omega_{\ell}(t, y) d t & =\left\langle K_{\ell}(x, \cdot), \Omega_{\ell}(\cdot, y)\right\rangle_{L_{2}(0, x)} \\
& =\sum_{n=0}^{\infty}\left\langle K_{\ell}(x, \cdot), p_{n}(x ; \cdot)\right\rangle_{L_{2}(0, x)}\left\langle p_{n}(x ; \cdot), \Omega_{\ell}(\cdot, y)\right\rangle_{L_{2}(0, x)} \\
& =\sum_{n=0}^{\infty} \alpha_{n}(x)\left\langle p_{n}(x ; \cdot), \Omega_{\ell}(\cdot, y)\right\rangle_{L_{2}(0, x)} \\
& =\sum_{n=0}^{\infty} \frac{\beta_{n}(x)}{\sqrt{4 n+2 \ell+3} \sqrt{x}} \int_{0}^{x} \frac{\sqrt{4 n+2 \ell+3}}{x^{\ell+3 / 2}} t^{\ell+1} P_{n}^{(\ell+1 / 2,0)}\left(1-\frac{2 t^{2}}{x^{2}}\right) \Omega_{\ell}(t, y) d t \\
& =\frac{1}{x^{\ell+2}} \sum_{n=0}^{\infty} \beta_{n}(x) \int_{0}^{x} t^{\ell+1} P_{n}^{(\ell+1 / 2,0)}\left(1-\frac{2 t^{2}}{x^{2}}\right) \Omega_{\ell}(t, y) d t .
\end{aligned}
$$

In order to proceed with the integral in (4.4), we need the following result [24]

$$
\int_{0}^{x} t^{\ell+1} P_{n}^{(\ell+1 / 2,0)}\left(1-\frac{2 t^{2}}{x^{2}}\right) b_{\ell}(\rho t) d t=x^{\ell+2} j_{\ell+2 n+1}(\rho x) .
$$

Hence

$$
\begin{aligned}
& \int_{0}^{x} t^{\ell+1} P_{n}^{(\ell+1 / 2,0)}\left(1-\frac{2 t^{2}}{x^{2}}\right) \Omega_{\ell}(t, y) d t \\
& \quad=x^{\ell+2}\left(\sum_{j=1}^{N} C_{j} j_{\ell+2 n+1}\left(i \tau_{j} x\right) b_{\ell}\left(i \tau_{j} y\right)+\frac{2}{\pi} \int_{0}^{\infty} j_{\ell+2 n+1}(\rho x) b_{\ell}(\rho y)\left(\left|F_{\ell}(\rho)\right|^{-2}-1\right) d \rho\right),
\end{aligned}
$$


and

$$
\begin{aligned}
& \int_{0}^{x} K_{\ell}(x, t) \Omega_{\ell}(t, y) d t \\
& \quad=\sum_{n=0}^{\infty} \beta_{n}(x)\left(\sum_{j=1}^{N} C_{j} j_{\ell+2 n+1}\left(i \tau_{j} x\right) b_{l}\left(i \tau_{j} y\right)+\frac{2}{\pi} \int_{0}^{\infty} j_{\ell+2 n+1}(\rho x) b_{\ell}(\rho y)\left(\left|F_{\ell}(\rho)\right|^{-2}-1\right) d \rho\right) .
\end{aligned}
$$

Thus, equation (3.9) can be written in the form

$$
\begin{aligned}
& \frac{y^{\ell+1}}{x^{\ell+2}} \sum_{n=0}^{\infty} \beta_{n}(x) P_{n}^{(\ell+1 / 2,0)}\left(1-\frac{2 y^{2}}{x^{2}}\right) \\
& +\sum_{n=0}^{\infty} \beta_{n}(x)\left(\sum_{j=1}^{N} C_{j} j_{\ell+2 n+1}\left(i \tau_{j} x\right) b_{\ell}\left(i \tau_{j} y\right)+\frac{2}{\pi} \int_{0}^{\infty} j_{\ell+2 n+1}(\rho x) b_{\ell}(\rho y)\left(\left|F_{\ell}(\rho)\right|^{-2}-1\right) d \rho\right) \\
& \quad=-\sum_{j=1}^{N} C_{j} b_{\ell}\left(i \tau_{j} x\right) b_{\ell}\left(i \tau_{j} y\right)-\frac{2}{\pi} \int_{0}^{\infty} b_{\ell}(\rho x) b_{\ell}(\rho y)\left(\left|F_{\ell}(\rho)\right|^{-2}-1\right) d \rho
\end{aligned}
$$

Multiplying (4.7) by $y^{\ell+1} P_{m}^{(\ell+1 / 2,0)}\left(1-\frac{2 y^{2}}{x^{2}}\right)$, integrating with respect to $y$ from 0 to $x$, and using (4.5) and (3.6) we obtain (4.3). The series in (4.3) converges again due to the general Parseval identity because it is a scalar product of the functions $K_{\ell}(x, t)$ and $\int_{0}^{x} \Omega_{\ell}(t, y) p_{m}(x ; t) d t$ in the space $L_{2}(0, x)$.

The functions $\frac{\beta_{m}(x)}{\sqrt{4 m+2 \ell+3} \sqrt{x}}$ are the Fourier coefficients of the function $K_{\ell}(x, \cdot)$ with respect to the system (3.7), see (3.8). It follows from (4.6) that the functions $\sqrt{4 m+2 \ell+3} \sqrt{x} \cdot B_{m}(x)$ are the Fourier coefficients of the function $-\Omega_{\ell}(x, \cdot)$ with respect to the system (3.7). Finally, multiplying (4.6) by $y^{\ell+1} P_{m}^{(\ell+1 / 2,0)}\left(1-\frac{2 y^{2}}{x^{2}}\right)$, integrating with respect to $y$ from 0 to $x$ and using (4.5) we obtain that

$$
x^{2 \ell+4} A_{n, m}(x)=\int_{0}^{x} \int_{0}^{x} t^{\ell+1} P_{n}^{(\ell+1 / 2,0)}\left(1-\frac{2 t^{2}}{x^{2}}\right) y^{\ell+1} P_{m}^{(\ell+1 / 2,0)}\left(1-\frac{2 y^{2}}{x^{2}}\right) \Omega_{\ell}(t, y) d t d y,
$$

or that

$$
\sqrt{4 n+2 \ell+3} \sqrt{4 m+2 \ell+3} \cdot x A_{m, n}(x)=\int_{0}^{x} \int_{0}^{x} p_{n}(t) p_{m}(y) \Omega_{\ell}(t, y) d t d y .
$$

The last equality means that the functions $\sqrt{4 n+2 \ell+3} \sqrt{4 m+2 \ell+3} \cdot x A_{m, n}(x)$ are the Fourier coefficients of the function $\Omega_{\ell}$ with respect to the system $p_{n} \times p_{m}$.

Hence for each fixed $x>0$ the infinite system (4.3) can be written as

$$
\xi_{j}-\lambda \sum_{k=0}^{\infty} a_{j k} \xi_{k}=b_{j}, \quad j=0,1, \ldots
$$

where $\lambda=-1$ and

$$
\xi_{j}=\frac{\beta_{j}(x)}{\sqrt{4 j+2 \ell+3} \sqrt{x}}, \quad b_{j}=\sqrt{4 j+2 \ell+3} \sqrt{x} \cdot B_{j}(x), \quad a_{j k}=\sqrt{4 j+2 \ell+3} \sqrt{4 k+2 \ell+3} \cdot x A_{j, k}(x) .
$$

The coefficient vectors satisfy $\left\{b_{j}\right\}_{j=0}^{\infty} \in \ell_{2},\left\{a_{j, k}\right\}_{j, k=0}^{\infty} \in \ell_{2} \otimes \ell_{2}$ and the unknown vector $\left\{\xi_{j}\right\}_{j=0}^{\infty}$ is sought to belong to $\ell_{2}$. The systems of such type, with coefficients from $\ell_{2}$, were studied in [14, Chapter $14, \S 3]$, and the following result follows immediately. 
Proposition 4.2. Let $x>0$ be fixed. Consider the system (4.3) truncated to $M+1$ equations, i.e., we consider $m, n \leq M$. Then for sufficiently large $M$ the truncated system has a unique solution which we denote by $\left\{\beta_{m}^{(M)}(x)\right\}_{m=0}^{M}$ and

$$
\sum_{m=0}^{M} \frac{\left|\beta_{m}(x)-\beta_{m}^{(M)}(x)\right|^{2}}{(4 m+2 \ell+3) x}+\sum_{m=M+1}^{\infty} \frac{\left|\beta_{m}(x)\right|^{2}}{(4 m+2 \ell+3) x} \rightarrow 0, \quad M \rightarrow \infty,
$$

from which it also follows that

$$
\beta_{0}^{(M)}(x) \rightarrow \beta_{0}(x), \quad M \rightarrow \infty .
$$

The same truncated system results from the application of the Bubnov-Galerkin procedure to the integral equation (3.9) with respect to the system (3.7), see [29, §14]. However, in our approach we do not need to solve the complete system, only the first function $\beta_{0}$ is necessary to recover the potential. Also we point out that the special form of the function system (3.7) allowed us to transform the scalar products arising in the Bubnov-Galerkin procedure into the form (4.1) and (4.2). As a consequence of the general theory presented in [29, §14] we obtain a stability result for the proposed method.

Let $I_{M}$ be the $(M+1) \times(M+1)$ identity matrix, $L_{M}=\left(a_{j k}\right)_{j, k=0}^{M}$ be the coefficient matrix of the truncated system and $R_{M}=\left(b_{j}\right)_{j=0}^{M}$ be the truncated right-hand side. Following [29, §9] consider a system (called non-exact system)

$$
\left(I_{M}+L_{M}+\Gamma_{M}\right) v=R_{M}+\delta_{M},
$$

where $\Gamma_{M}$ is an $(M+1) \times(M+1)$ matrix representing errors in the coefficients $a_{j k}$, and $\delta_{M}$ is a column-vector representing errors in the coefficients $b_{j}$. Let $U_{M}$ denote the solution of the exact truncated system (with $\Gamma_{M}=0$ and $\delta_{M}=0$ ) and $V_{M}$ the solution of the non-exact system. Note that $U_{M}=\left\{\frac{\beta_{m}^{M}(x)}{\sqrt{4 m+2 \ell+3} \sqrt{x}}\right\}_{m=0}^{M}$, see Proposition 4.2. The solution of the Bubnov-Galerkin procedure is called stable if there exist constants $c_{1}, c_{2}$ and $r$ independent of $M$ such that for $\left\|\Gamma_{M}\right\| \leq r$ and arbitrary $\delta_{M}$ the non-exact system is solvable and the following inequality holds

$$
\left\|U_{M}-V_{M}\right\| \leq c_{1}\left\|\Gamma_{M}\right\|+c_{2}\left\|\delta_{M}\right\| .
$$

From [29, Theorems 14.1 and 14.2] the following result follows.

Proposition 4.3. The approximate solution $\left\{\frac{\beta_{m}^{M}(x)}{\sqrt{4 m+2 \ell+3} \sqrt{x}}\right\}_{m=0}^{M}$ of system (4.8) is stable. Moreover, the condition numbers of the coefficient matrices $I_{M}+L_{M}$ are bounded.

This result allows one to recover the potential from inexact to a certain point or noisy scattering data.

\section{$5 \quad$ Numerical implementation}

\subsection{General scheme}

Theorem 4.1 and Proposition 4.2 lead to a direct and simple method for solving the inverse quantum scattering problem. 
1. Given the Jost function, the eigenvalues and the norming constants. Choose a number of equations $M+1$, so that the truncated system

$$
\frac{\beta_{m}(x)}{(4 m+2 \ell+3) x}+\sum_{n=0}^{M} \beta_{n}(x) A_{m, n}(x)=B_{m}(x), \quad \text { for all } m=0, \ldots, M
$$

is to be solved.

2. Compute $B_{m}(x)$ and $A_{m, n}(x)$ according to the formulas (4.2) and (4.1).

3. Solve the system (5.1) to find $\beta_{0}(x)$.

4. Compute $q$ with the aid of (3.5) or by computing first the particular solution $u_{\ell, 0}$ using (3.4).

Remark 5.1. Since the condition numbers of truncated systems (4.8) are bounded, see Proposition 4.3, it may be worth converting the system (5.1) into the truncated system of the form (4.8) for large values of $M$.

\subsection{On calculation of the integrals}

Calculation of the integrals in (4.1) and (4.2) is one of the key steps in the proposed method. Since for $\rho \in \mathbb{R}$

$$
\left|j_{\nu}(\rho x)\right|=\frac{\cos \left(\rho x-\frac{1}{2} \pi \nu-\frac{\pi}{4}\right)}{|\rho x|}+O\left(\frac{1}{|\rho x|^{2}}\right), \quad|\rho| \rightarrow \infty,
$$

see [1, (9.2.1)] and

$$
\left|F_{\ell}(\rho)\right|^{-2}-1=\frac{1}{4 \rho^{2}}\left(\int_{0}^{\infty} q(x) d x\right)^{2}+O\left(\frac{1}{\rho^{2}}\right)=O\left(\frac{1}{\rho^{2}}\right), \quad|\rho| \rightarrow \infty,
$$

see (2.5), we have

$$
\left|j_{\ell+2 n+1}(\rho x) j_{\ell+2 m+1}(\rho x)\left(\left|F_{\ell}(\rho)\right|^{-2}-1\right)\right| \leq \frac{c_{1}}{x^{2} \rho^{4}}
$$

and

$$
\left|b_{\ell}(\rho x) j_{\ell+2 m+1}(\rho x)\left(\left|F_{\ell}(\rho)\right|^{-2}-1\right)\right| \leq \frac{c_{2}}{x \rho^{3}}, \quad \rho \rightarrow+\infty .
$$

As one can see, the integral in (4.2) can converge slowly. The convergence can be improved to some extent subtracting leading term and integrating it explicitly. Note that due to (5.2),

$$
\rho^{2}\left(\left|F_{\ell}(\rho)\right|^{-2}-1\right)=O(1), \quad|\rho| \rightarrow \infty,
$$

that is, a bounded term. Numerical experiments suggest that this bounded term is a sum of a constant, an oscillating function and an $o(1)$ function. The value of the constant, which we will denote by $\tilde{F}_{\ell}$, can be easily estimated numerically. For example, one can compute the expression (5.3) for some set of points $\left\{\rho_{k}\right\}_{k=0}^{K}$ and take for $\tilde{F}_{\ell}$ an average of the obtained values. See Figure 1 for an illustration.

Note also that (see [31, 2.12.31.2])

$$
\int_{0}^{\infty} \frac{j_{\ell+2 n+1}(\rho x) j_{\ell+2 m+1}(\rho x)}{\rho^{2}} d \rho= \begin{cases}\frac{\pi x}{4(\ell+2 n+1 / 2)_{3}}, & \text { if } n=m, \\ \frac{\pi x}{8(\ell+n+m+1 / 2)_{3}}, & \text { if } n=m \pm 1 \\ 0, & \text { if }|n-m| \geq 2\end{cases}
$$


and

$$
\int_{0}^{\infty} \frac{b_{\ell}(\rho x) j_{\ell+2 m+1}(\rho x)}{\rho^{2}} d \rho= \begin{cases}\frac{\pi x}{2(\ell+1 / 2)_{2}}, & \text { if } m=0, \\ 0, & \text { if } m>0,\end{cases}
$$

where $(x)_{n}$ stands for the Pochhammer symbol. Hence instead of computing integrals (4.1) and (4.2) one can compute the integrals

$$
\int_{0}^{\infty} j_{\ell+2 n+1}(\rho x) j_{\ell+2 m+1}(\rho x)\left(\left|F_{\ell}(\rho)\right|^{-2}-1-\frac{\tilde{F}_{\ell}}{\rho^{2}}\right) d \rho
$$

and

$$
\int_{0}^{\infty} b_{\ell}(\rho x) j_{\ell+2 m+1}(\rho x)\left(\left|F_{\ell}(\rho)\right|^{-2}-1-\frac{\tilde{F}_{\ell}}{\rho^{2}}\right) d \rho
$$

and afterwards add expressions (5.4) and (5.5) multiplied by $\tilde{F}_{\ell}$. If the integrals are truncated and computed on a segment $[0, K]$, the proposed modification leads to a more accurate result due to the integral tail taken into account (the oscillating and $o(1)$ parts in (5.3) are expected to result in smaller values in comparison with the part given by the constant $\tilde{F}_{\ell}$. . We would like to mention that the proposed modification is nothing more than an adaptation of the method presented in [30, (9.98)] with first two terms taken into account. Note also that in the case $\ell=-1 / 2$ the expression (5.4) can not be used for $n=m=0$, and the expression (5.5) for $m=0$ (due to the divergence at the origin). One should use directly expressions (4.1) and (4.2). For $\ell<0$ the integrals (5.6) and (5.7) possess integrable singularity at the origin.

In the present work we opted out of applying special methods for calculating oscillatory integrals and the Hankel transform in particular (see, e.g., [30], 39] and references therein). The main reason is that the function $F_{\ell}$ oscillates a lot even for simplest potentials, see Figure 1, For that reason we are not expecting a simple approximation of the term $\left|F_{\ell}(\rho)\right|^{-2}-1$ to be possible and decided to leave a detailed study for a separate work.

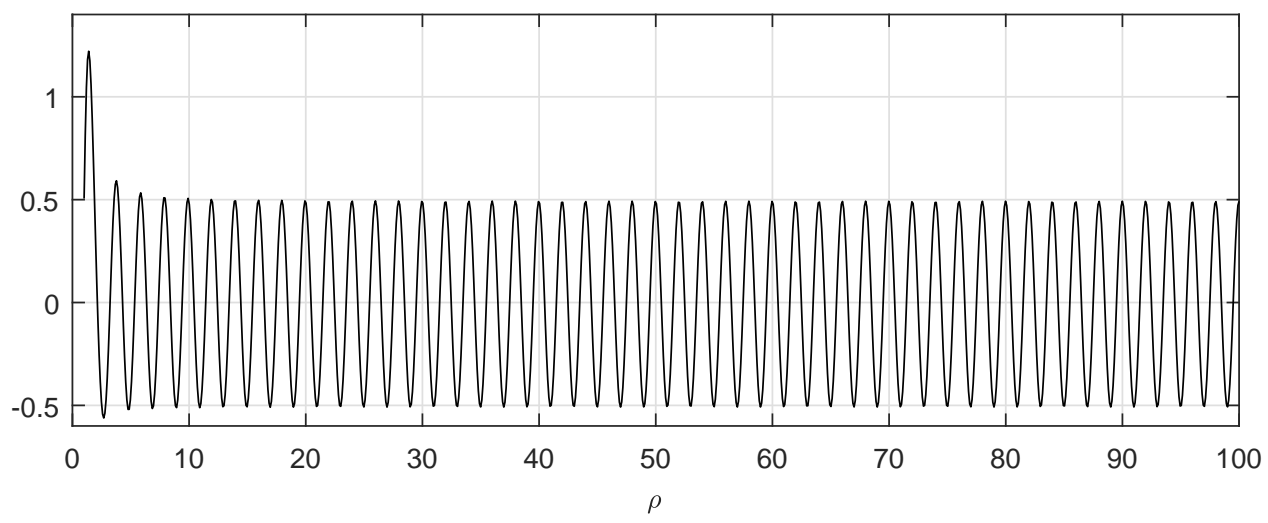

Figure 1: Plot of the function $\rho^{2}\left(\left|F_{\ell}(\rho)\right|^{-2}-1-\frac{\tilde{F}_{\ell}}{\rho^{2}}\right)$ for the square well potential from Example 2.1 with $\ell=1, Q=1$ and $R=\pi / 2$. The parameter $\tilde{F}_{\ell}$ is estimated numerically to be 1.5079 .

\subsection{Numerical examples}

The numerical illustrations presented below were obtained in Matlab2017. For the numerical integration on step 2 a sufficiently large interval was chosen and the Matlab routine trapz was used. On the last step, for recovering $q$ we used (3.5). Here the differentiation was performed by representing 
the computed function $\beta_{0}(x)$ in the form of a spline with the aid of the Matlab routine spapi with a posterior differentiation with the Matlab command fnder.

Example 5.2. Consider the potential (2.7) with $\ell=2, Q=1$ and $R=\pi / 2$. On Figure 2 the recovered potential (on the left) and its absolute error (on the right) are shown in the cases $M=0, M=1, M=4$ and $M=9$ that corresponds to 1, 2, 5 and 10 equations in the truncated system (5.1), respectively. Thus, a very reduced number of equations from the system (5.1) is sufficient even in the case of a discontinuous potential. For the numerical integration we have used the interval $\rho \in[0,5000]$. However it should be mentioned that such a large interval was used only to demonstrate that the method can recover smooth potentials with a 10 decimal digits accuracy. Reducing the integration to the interval $[0,100]$ and taking the step-size of $1 / 10$ for the trapz function still allowed us to recover the potential with 4-5 decimal figures.
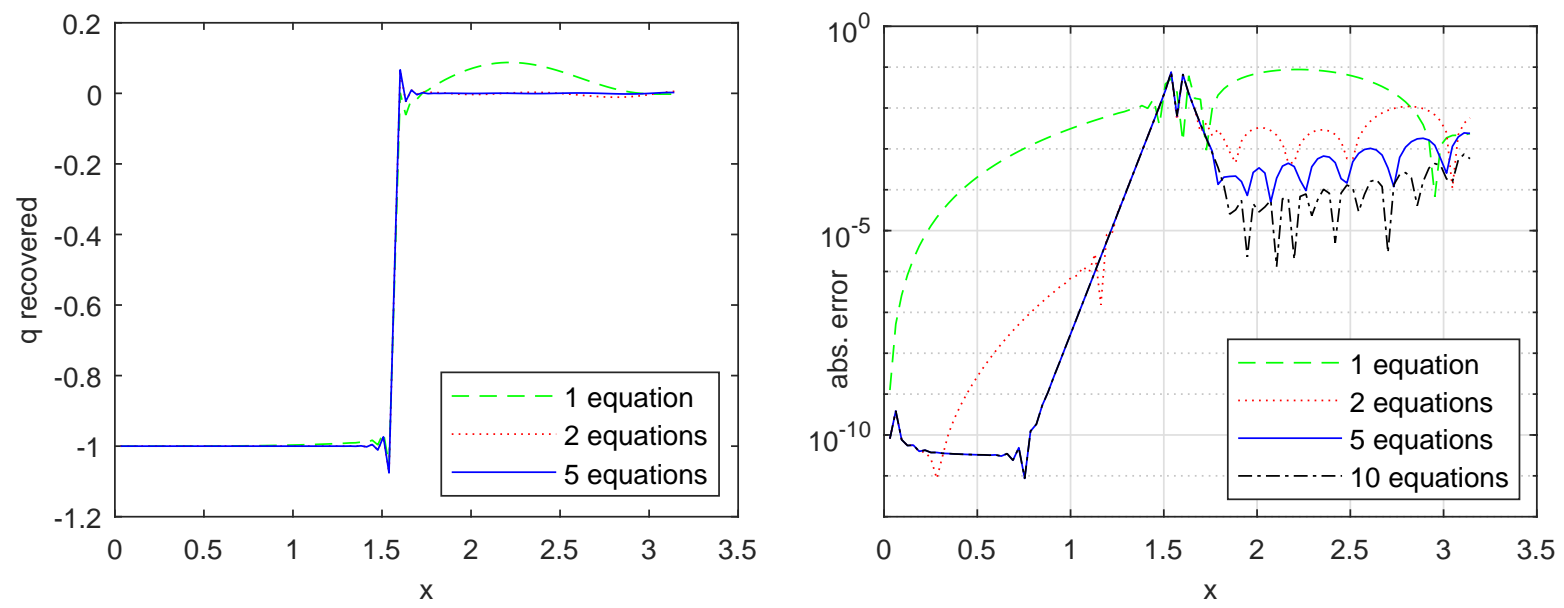

Figure 2: On the left: the square well potential from Example 5.2 with $\ell=2$ recovered on the interval $(0, \pi]$ with $M=0, M=1$ and $M=4$ that corresponds to 1,2 and 5 equations in the truncated system (5.1), respectively. On the right: absolute error of the recovered potential for $M \in\{0,1,4,9\}$ corresponding to $1,2,5$ and 10 equations in the truncated system (5.1), respectively.

Note that the error increase in the recovered potential closer to the discontinuity point $x=\pi / 2$ is due to the error propagation in the spline interpolation procedure. Indeed, on Figure 3, left plot, we show the absolute error of the recovered coefficient $\beta_{0}$. As one can appreciate, the error remains small almost up to the discontinuity point $x=\pi / 2$. So one can expect that applying numerical differentiation without using values of $\beta_{0}$ from both sides of the discontinuity point, e.g., the finite difference or constructing a spline using the data from $[0, \pi / 2]$ only, can reduce the error for values of $x$ close to $\pi / 2$. Indeed, on Figure 3, right plot, we show the error of the recovered potential when the coefficient $\beta_{0}$ was approximated by a spline separately on $[0, \pi / 2]$ and on $[\pi / 2, \pi]$. One can appreciate a higher accuracy close to $x=\pi / 2$.

Example 5.3. The method gives excellent results for negative values of $\ell$ and for larger values of $\ell$ as well. Let us consider the same potential as in Example 5.2 but for $\ell=-1 / 2$ and $\ell=e^{3}$.

Note that for $\ell=-1 / 2$ the problem possesses an eigenvalue. We used the method from [24] to find its value, $\rho_{1}^{2} \approx-0.258265599397038$, with a corresponding norming constant $c_{1} \approx 0.469060824384319$.

On Figure 4 we show the absolute errors of the recovered potentials.

Example 5.4. Let us consider the equation with the Hulthén effective potential

$$
L_{1} u:=-u^{\prime \prime}+\left(\ell(\ell+1)\left(\frac{\delta}{1-e^{-\delta x}}\right)^{2} e^{-\delta x}-\frac{2 \delta e^{-\delta x}}{1-e^{-\delta x}}\right) u=\rho^{2} u, \quad x>0 .
$$



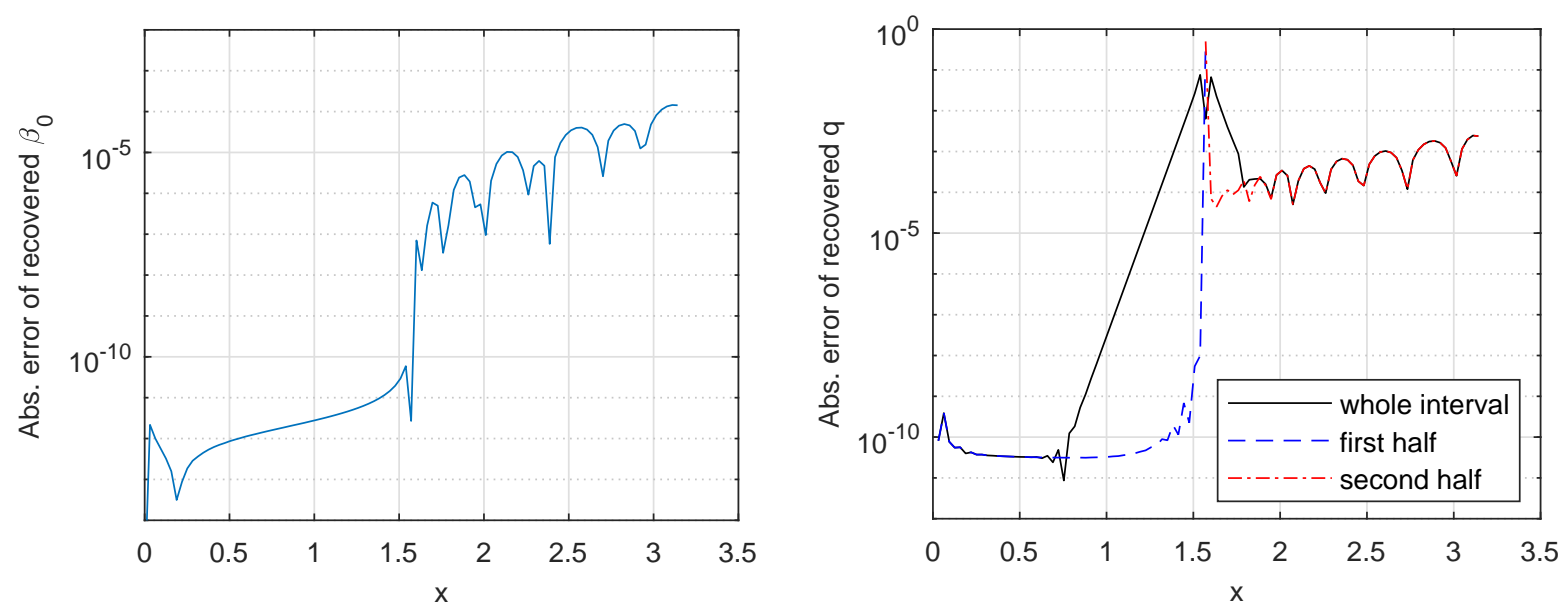

Figure 3: On the left: absolute error of the recovered coefficient $\beta_{0}$ for the square well potential from Example 5.2 with $\ell=2, M=4$ on the interval $(0, \pi]$. On the right: absolute error of the recovered potential depending on the choise of the interval used for spline interpolation of the coefficient $\beta_{0}$ and differentiation.

Here $0<\delta<1$.

The Hulthén potential $q_{H}(r)=-\frac{2 \delta e^{-\delta x}}{1-e^{-\delta x}}$ is known as a potential providing a better approximation to the screened Coulomb (Yukawa) potential $q_{s c}(r)=-\frac{2 e^{-\delta r}}{r}$ than the ordinary Coulomb potential $-\frac{2}{r}$, see, e.g., [27], [11. However, it can be exactly solved only for zero angular momentum, i.e., for $\ell=0$. Greene and Aldrich [11] considered the Hulthén effective potential as an exactly solvable approximation for all values of $\ell$. Equation (5.8) can be transformed into the form (2.1) if one considers

$$
q(x)=\ell(\ell+1)\left(\frac{\delta}{1-e^{-\delta x}}\right)^{2} e^{-\delta x}-\frac{\ell(\ell+1)}{x^{2}}-\frac{2 \delta e^{-\delta x}}{1-e^{-\delta x}} .
$$

Note that $q(x) \sim-\frac{2}{x}+\frac{\delta \ell(\ell+1)}{x}$ as $x \rightarrow 0$ and $q(x) \sim-\frac{\ell(\ell+1)}{x^{2}}$ as $x \rightarrow \infty$, so the potential $q$ does not satisfy the condition (2.2). Nevertheless, the spectral problem for the original equation (5.8) possesses at most a finite number of negative eigenvalues, see, e.g., [35], so the corresponding quantum scattering problem can be solved by the same method, see [5].

To simplify the consideration below in what follows we assume that $2 \ell \notin \mathbb{Z}$. Then the general solution of (5.8) has the form

$$
\begin{aligned}
u(x)= & A y^{-\ell} e^{i \rho x}{ }_{2} F_{1}\left(-\ell-a_{\rho}-s_{\rho},-\ell-a_{\rho}+s_{\rho} ;-2 \ell ; y\right) \\
& +B y^{\ell+1} e_{2}^{i \rho x}{ }_{2} F_{1}\left(\ell+1-a_{\rho}-s_{\rho}, \ell+1-a_{\rho}+s_{\rho} ; 2 \ell+2 ; y\right),
\end{aligned}
$$

where $y=1-e^{-\delta x}, a_{\rho}=i \frac{\rho}{\delta}$ and $s_{\rho}=\frac{\sqrt{2 \delta-\rho^{2}}}{\delta}$. This expression was obtained solving transformed equation (7) from [11] using Wolfram Mathematica 10.

Note that $y \rightarrow 0$ as $x \rightarrow 0$, hence the regular solution of (5.8) has the form

$$
\varphi_{\ell}(\rho, x)=\frac{\left(1-e^{-\delta x}\right)^{\ell+1} e^{i \rho x}}{\delta^{\ell+1}(2 \ell+1) ! !}{ }_{2} F_{1}\left(\ell+1-a_{\rho}-s_{\rho}, \ell+1-a_{\rho}+s_{\rho} ; 2 \ell+2 ; 1-e^{-\delta x}\right) .
$$

On the other hand, $y \rightarrow 1$ as $x \rightarrow+\infty$, so the values of the hypergeometric functions in (5.9) are not defined by their series expansions and to find the Jost solution we need to apply the following analytic 

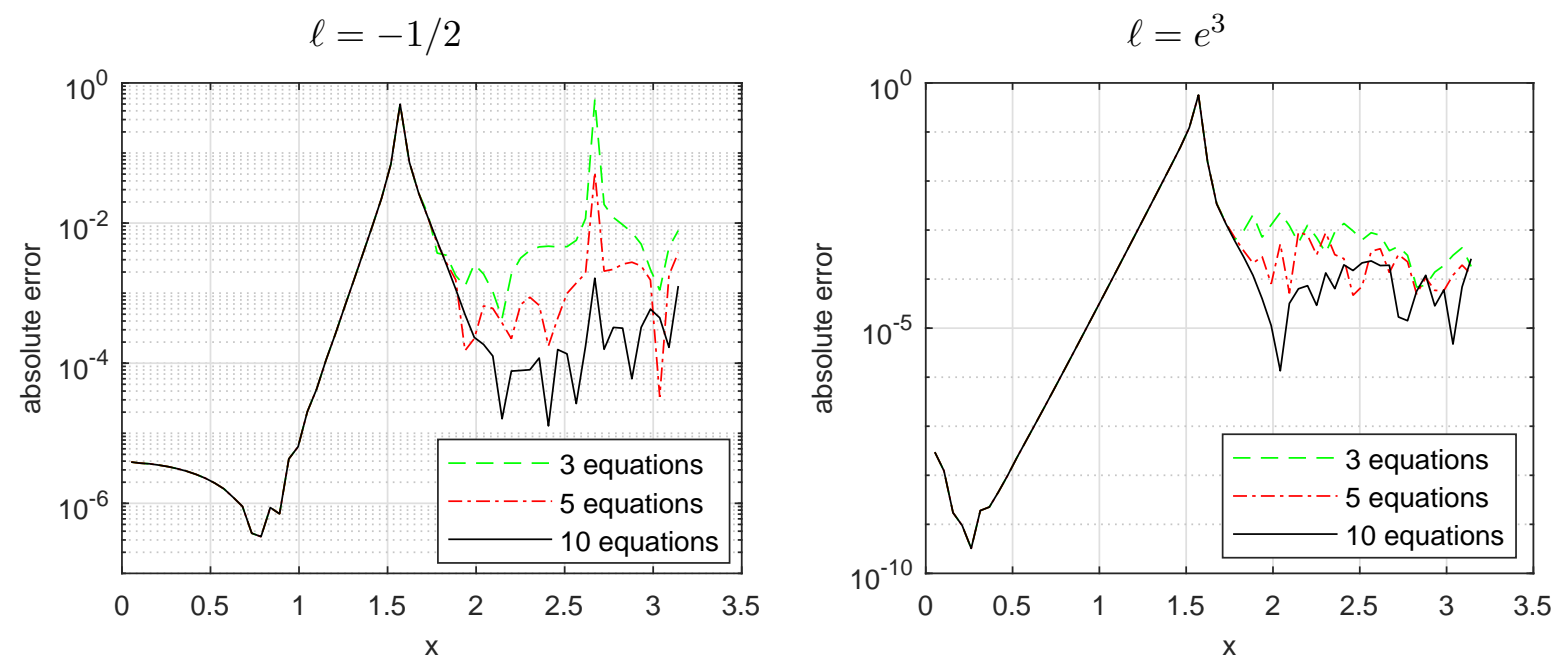

Figure 4: Absolute errors of the recovered square well potential from Example 5.3 having $\ell=-1 / 2$ (on the left) and $\ell=e^{3}$ (on the right). The potential was recovered on the interval $(0, \pi]$ and 3,5 or 10 equations were used in the truncated system (5.1).

continuation [9, (2.10.1)]

$$
\begin{aligned}
{ }_{2} F_{1}(a, b ; c ; z)= & \frac{\Gamma(c) \Gamma(c-a-b)}{\Gamma(c-a) \Gamma(c-b)}{ }_{2} F_{1}(a, b ; a+b-c+1 ; 1-z) \\
& +\frac{\Gamma(c) \Gamma(a+b-c)}{\Gamma(a) \Gamma(b)}(1-z)^{c-a-b}{ }_{2} F_{1}(c-a, c-b ; c-a-b+1 ; 1-z) .
\end{aligned}
$$

Then

$$
\begin{aligned}
u(x) & =A \frac{y^{-\ell} e^{i \rho x} \Gamma(-2 \ell) \Gamma\left(2 a_{\rho}\right)}{\Gamma\left(-\ell+a_{\rho}+s_{\rho}\right) \Gamma\left(-\ell+a_{\rho}-s_{\rho}\right)}{ }_{2} F_{1}\left(-\ell-a_{\rho}-s_{\rho},-\ell-a_{\rho}+s_{\rho} ; 1-2 a_{\rho} ; e^{-\delta x}\right) \\
& +A \frac{y^{-\ell} e^{-i \rho x} \Gamma(-2 \ell) \Gamma\left(-2 a_{\rho}\right)}{\Gamma\left(-\ell-a_{\rho}-s_{\rho}\right) \Gamma\left(-\ell-a_{\rho}+s_{\rho}\right)}{ }_{2} F_{1}\left(-\ell+a_{\rho}+s_{\rho},-\ell+a_{\rho}-s_{\rho} ; 1+2 a_{\rho} ; e^{-\delta x}\right) \\
& +B \frac{y^{\ell+1} e^{i \rho x} \Gamma(2 \ell+2) \Gamma\left(2 a_{\rho}\right)}{\Gamma\left(\ell+1+a_{\rho}+s_{\rho}\right) \Gamma\left(\ell+1+a_{\rho}-s_{\rho}\right)}{ }_{2} F_{1}\left(\ell+1-a_{\rho}-s_{\rho}, \ell+1-a_{\rho}+s_{\rho} ; 1-2 a_{\rho} ; e^{-\delta x}\right) \\
& +B \frac{y^{\ell+1} e^{-i \rho x} \Gamma(2 \ell+2) \Gamma\left(-2 a_{\rho}\right)}{\Gamma\left(\ell+1-a_{\rho}-s_{\rho}\right) \Gamma\left(\ell+1-a_{\rho}+s_{\rho}\right)}{ }_{2} F_{1}\left(\ell+1+a_{\rho}+s_{\rho}, \ell+1+a_{\rho}-s_{\rho} ; 1+2 a_{\rho} ; e^{-\delta x}\right) .
\end{aligned}
$$

The first and the third terms behave like constant by $e^{i \rho x}$ when $x \rightarrow \infty$, while the second and the forth terms behave like constant by $e^{-i \rho x}$ when $x \rightarrow \infty$. Hence for the solution $u$ to be the Jost solution, the coefficients $A$ and $B$ have to satisfy the following system

$$
\begin{aligned}
& A \cdot \frac{\Gamma(-2 \ell) \Gamma\left(2 a_{\rho}\right)}{\Gamma\left(-\ell+a_{\rho}+s_{\rho}\right) \Gamma\left(-\ell+a_{\rho}-s_{\rho}\right)}+B \cdot \frac{\Gamma(2 \ell+2) \Gamma\left(2 a_{\rho}\right)}{\Gamma\left(\ell+1+a_{\rho}+s_{\rho}\right) \Gamma\left(\ell+1+a_{\rho}-s_{\rho}\right)}=e^{i \pi \ell / 2} \\
& A \cdot \frac{\Gamma(-2 \ell) \Gamma\left(-2 a_{\rho}\right)}{\Gamma\left(-\ell-a_{\rho}-s_{\rho}\right) \Gamma\left(-\ell-a_{\rho}+s_{\rho}\right)}+B \cdot \frac{\Gamma(2 \ell+2) \Gamma\left(-2 a_{\rho}\right)}{\Gamma\left(\ell+1-a_{\rho}-s_{\rho}\right) \Gamma\left(\ell+1-a_{\rho}+s_{\rho}\right)}=0 .
\end{aligned}
$$


Solving this system and using Euler's reflection formula $\Gamma(1-z) \Gamma(z)=\frac{\pi}{\sin \pi z}$ we obtain that

$$
\begin{aligned}
& A=e^{i \pi \ell / 2} \frac{\Gamma\left(1-2 a_{\rho}\right) \Gamma(2 \ell+1)}{\Gamma\left(\ell+1-a_{\rho}+s_{\rho}\right) \Gamma\left(\ell+1-a_{\rho}-s_{\rho}\right)}, \\
& B=-e^{i \pi \ell / 2} \frac{\Gamma(-2 \ell) \Gamma\left(1-2 a_{\rho}\right)}{(2 \ell+1) \Gamma\left(-\ell-a_{\rho}+s_{\rho}\right) \Gamma\left(-\ell-a_{\rho}-s_{\rho}\right)} .
\end{aligned}
$$

The Jost function is given by

$$
F_{\ell}(\rho)=\lim _{x \rightarrow 0} \frac{(-\rho x)^{\ell}}{(2 \ell-1) ! !} \frac{A e^{i \rho x}}{\left(1-e^{-\delta x}\right)^{\ell}}=\frac{A}{(2 \ell-1) ! !}\left(-\frac{\rho}{\delta}\right)^{\ell}
$$

hence

$$
F_{\ell}(\rho)=\frac{e^{i \pi \ell / 2}}{(2 \ell-1) ! !} \frac{\Gamma\left(1-2 i \frac{\rho}{\delta}\right) \Gamma(2 \ell+1)}{\Gamma\left(\ell+1-i \frac{\rho}{\delta}+\frac{\sqrt{2 \delta-\rho^{2}}}{\delta}\right) \Gamma\left(\ell+1-i \frac{\rho}{\delta}-\frac{\sqrt{2 \delta-\rho^{2}}}{\delta}\right)}\left(-\frac{\rho}{\delta}\right)^{\ell} .
$$

Note that this expression is also well defined for values $\ell$ satisfying $2 \ell \in \mathbb{N}$.

The eigenvalues $\rho_{j}=i \tau_{j}, \tau_{j} \geq 0$ correspond to zeros of the function $F_{\ell}$. One can easily see that all such zeros coincide with the values of $\rho$ for which $\Gamma\left(\ell+1-i \frac{\rho}{\delta}-\frac{\sqrt{2 \delta-\rho^{2}}}{\delta}\right)=\infty$, i.e., when the argument of the gamma function is a non-positive integer, which is equivalent to the equation

$$
\ell+1+\frac{\tau}{\delta}-\frac{\sqrt{2 \delta+\tau^{2}}}{\delta}=-m, \quad m \in \mathbb{N}_{0} .
$$

Squaring the equation we find that

or

$$
(\ell+1+m)^{2}+\frac{\tau^{2}}{\delta^{2}}+2 \frac{\tau}{\delta}(\ell+1+m)=\frac{2}{\delta}+\frac{\tau^{2}}{\delta^{2}}
$$

$$
\tau=\frac{1}{\ell+1+m}-\frac{\delta}{2}(\ell+1+m), \quad m \in \mathbb{N}_{0}
$$

Recalling that $\tau$ must be non-negative, we find that the set of eigenvalues is given by

$$
\tau_{j}=\frac{1}{\ell+j}-\frac{\delta}{2}(\ell+j), \quad j=1, \ldots,\left[\sqrt{\frac{2}{\delta}}-\ell\right],
$$

where [·] is the integer part function. The corresponding eigenfunctions are given by

$$
\varphi_{\ell}\left(i \tau_{j}, x\right)=\frac{\left(1-e^{-\delta x}\right)^{\ell+1} e^{i \rho x}}{\delta^{\ell+1}(2 \ell+1) ! !}{ }_{2} F_{1}\left(-j+1, \ell+1+\frac{2}{\delta(\ell+1)} ; 2 \ell+2 ; 1-e^{-\delta x}\right) .
$$

Note that the first argument of the hypergeometric function is a non-positive integer, so the hypergeometric function reduces to a polynomial. The corresponding norming constants can be easily obtained numerically.

On Figure 5 we show the recovered potential and the absolute error. In this example the function $\left|F_{\ell}(\rho)\right|^{-2}-1$ decays as $1 / \rho$, and not as $1 / \rho^{2}$ as was considered in Subsection 5.2. However, a similar procedure was implemented to improve the computation of the integrals. The interval $\rho \in[0,1000]$ was used for numerical integration.

Proposition 4.3 states that the condition numbers of the matrices arising in the process remain bounded and that the method is stable for a small noise. We illustrate these statements by Figure 6 . On the left we show the smallest and the largest eigenvalues of the coefficient matrix of the truncated system (4.8) as the function of the truncation parameter $M$. As one can see, the condition number, which is equal to the quotient of these eigenvalues, remains bounded independently of the number of equations used. On the right we show the potential recovered from the noisy data $\left\{\tau_{j}, c_{j}\right\}_{j=1}^{4}$ and $F_{\ell}(\rho), \rho \in\left\{\frac{k}{10}\right\}_{k=0}^{1000}, 10 \%$ noise was added to all the values. 

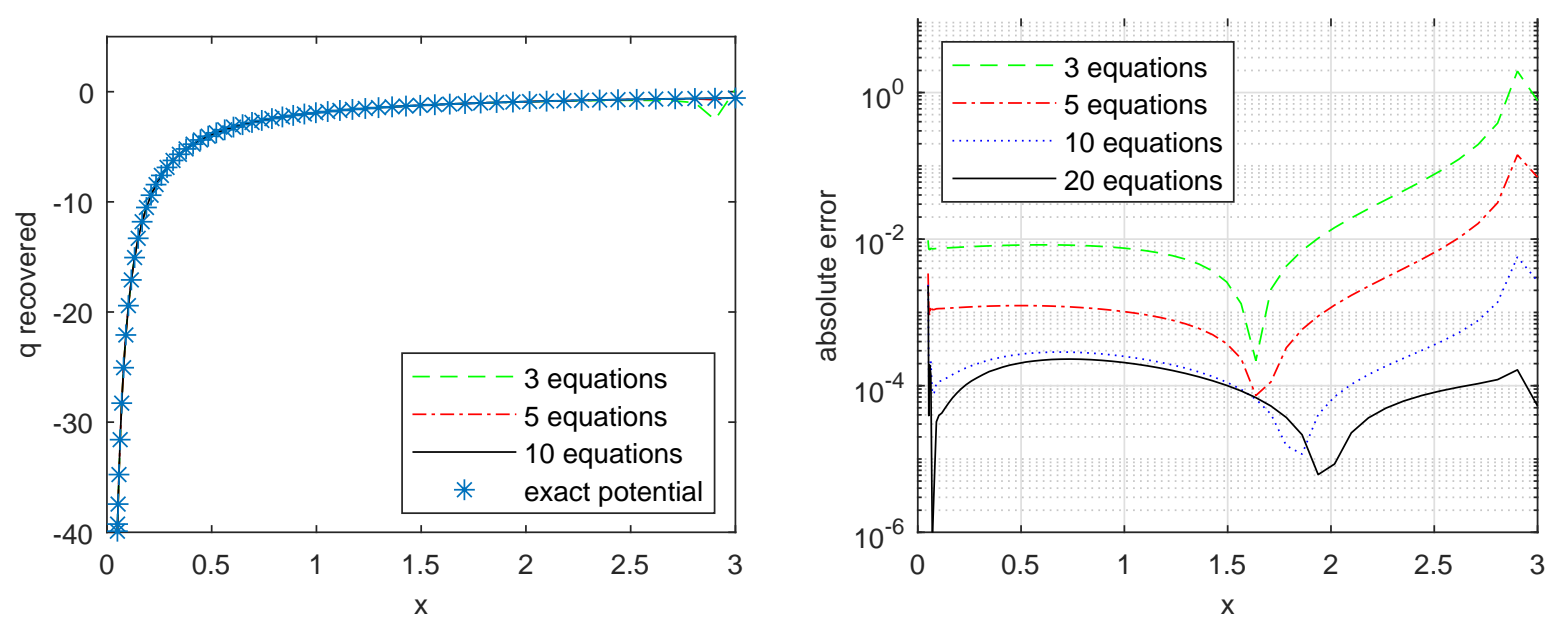

Figure 5: Recovered Hulthén effective potential from Example 5.4 with $\ell=1 / 3$ and $\delta=1 / 10$ (on the left) and the absolute error (on the right). The potential was recovered on the interval $\left[\frac{1}{20}, 3\right]$ using up to 20 equations in the truncated system (5.1).

\section{Conclusions}

A direct and simple method for solving the inverse quantum scattering problem for an arbitrary angular momentum $\ell \geq-1 / 2$ is presented. Numerical solution of the problem reduces to the solution of a system of linear algebraic equations from which the first component of the solution vector is sufficient for recovering the potential. Numerical results reveal a remarkable accuracy, stability and a fast convergence of the method.

\section{A On the asymptotic behaviour of the function $F_{\ell}$}

According to [18, Lemma B.5] the function $F_{\ell}$ admits the following integral representation

$$
F_{\ell}(\rho)=1+\int_{0}^{\infty} \psi_{\ell}^{0}(\rho, x) \varphi_{\ell}(\rho, x) q(x) d x,
$$

where $\varphi_{\ell}$ is the regular solution of (2.1) considered in Section 2 and $\psi_{\ell}^{0}$ denotes the Jost solution of (2.1) with $q \equiv 0$. We also denote the regular solution for $q \equiv 0$ by $\varphi_{\ell}^{0}$.

First, we recall some estimates from [18] and [19]. The solutions $\varphi_{\ell}^{0}$ and $\psi_{\ell}^{0}$ of the unperturbed equation are given by

$$
\begin{aligned}
& \varphi_{\ell}^{0}(\rho, x)=\rho^{-\ell-1 / 2} \sqrt{\frac{\pi x}{2}} J_{\ell+1 / 2}(\rho x), \\
& \psi_{\ell}^{0}(\rho, x)=i \rho^{\ell+1 / 2} \sqrt{\frac{\pi x}{2}} H_{\ell+1 / 2}^{(1)}(\rho x) .
\end{aligned}
$$

Here $H_{\ell+1 / 2}^{0}$ is the Hankel function of the first kind. The following estimates hold for $\rho \in \mathbb{R}, \ell>-1 / 2$

$$
\left|\varphi_{\ell}^{0}(\rho, x)\right| \leq C\left(\frac{x}{1+|\rho| x}\right)^{\ell+1}, \quad\left|\psi_{\ell}^{0}(\rho, x)\right| \leq C\left(\frac{x}{1+|\rho| x}\right)^{-\ell} .
$$

For $\ell=-1 / 2$ the first estimate remains valid, and the second changes to

$$
\left|\psi_{-1 / 2}^{0}(\rho, x)\right| \leq C\left(\frac{x}{1+|\rho| x}\right)^{1 / 2}\left(1-\log \frac{|\rho| x}{1+|\rho| x}\right) .
$$



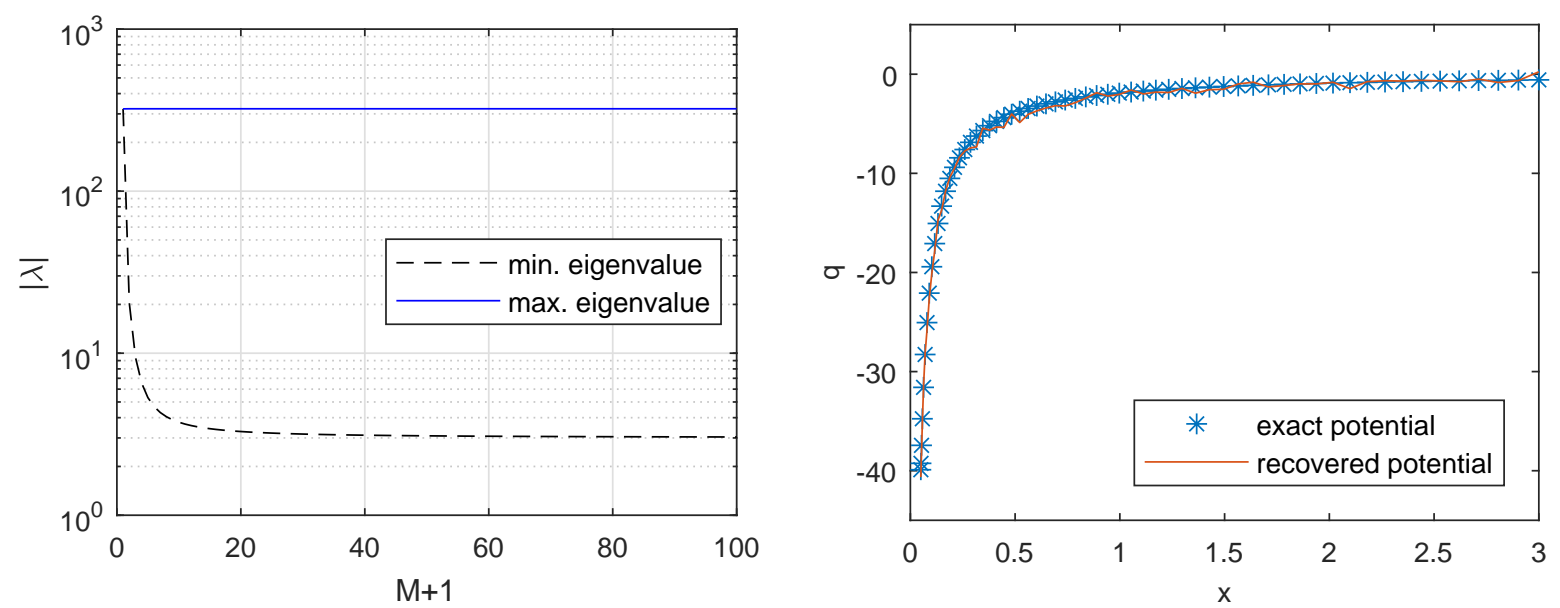

Figure 6: The Hulthén effective potential from Example 5.4 having $\ell=1 / 3$ and $\delta=1 / 10$ is considered. On the left: the smallest and the largest eigenvalues of the coefficient matrix of the truncated system for $x=3$ as the function of $M$. On the right: potential recovered from the noisy data.

Considering the difference between the regular solutions $\varphi_{\ell}$ and $\varphi_{\ell}^{0}$, the following estimate follows from [17, (2.21) and (2.23)] and [18, Lemma B.2]

$$
\begin{aligned}
\left|\varphi_{\ell}(\rho, x)-\varphi_{\ell}^{0}(\rho, x)\right| & \leq \sum_{n=1}^{\infty} \frac{C^{n+1}}{n !}\left(\frac{x}{1+|\rho| x}\right)^{\ell+1} e^{|\operatorname{Im} \rho| x}\left(\int_{0}^{x} \frac{y|\bar{q}(y)|}{1+|\rho| y} d y\right)^{n} \\
& =C\left(\frac{x}{1+|\rho| x}\right)^{\ell+1} e^{|\operatorname{Im} \rho| x}\left(\exp \left(C \int_{0}^{x} \frac{y|\bar{q}(y)|}{1+|\rho| y} d y\right)-1\right),
\end{aligned}
$$

here $\bar{q}(x)=q(x)$ if $\ell>-1 / 2$ and $\bar{q}(x)=\left(1-\log \left(\frac{x}{1+x}\right)\right) q(x)$ if $\ell=-1 / 2$.

For large values of arguments the functions $\varphi_{\ell}^{0}$ and $\psi_{\ell}^{0}$ can be approximated using the asymptotic formulas for the functions $J_{\nu}$ and $H_{\nu}^{(1)}$. We have (see [1, (9.2.5)-(9.2.10)] for $z \in \mathbb{R}$

$$
J_{\nu}(z)=\sqrt{\frac{2}{\pi z}}(P(\nu, z) \cos \chi-Q(\nu, z) \sin \chi), \quad H_{\nu}^{(1)}(z)=\sqrt{\frac{2}{\pi z}}(P(\nu, z)+i Q(\nu, z)) e^{i \chi}
$$

where $\chi=z-\left(\frac{\nu}{2}+\frac{1}{4}\right) \pi$ and

$$
\begin{aligned}
& P(\nu, z)=\sum_{k=0}^{[\nu / 2+3 / 4]}(-1)^{k} \frac{(\nu, 2 k)}{(2 z)^{2 k}}+\theta_{1}(z) \frac{(\nu, 2[\nu / 2+7 / 4])}{(2 z)^{2[\nu / 2+7 / 4]}} \\
& Q(\nu, z)=\sum_{k=0}^{[\nu / 2+1 / 4]}(-1)^{k} \frac{(\nu, 2 k+1)}{(2 z)^{2 k+1}}+\theta_{2}(z) \frac{(\nu, 2[\nu / 2+5 / 4]+1)}{(2 z)^{2[\nu / 2+5 / 4]+1}},
\end{aligned}
$$

with $\left|\theta_{1,2}\right| \leq 1$. From (A.7) (A.9) it follows that for all $z \geq 1$

$$
\begin{aligned}
J_{\nu}(z) & =\sqrt{\frac{2}{\pi z}}\left(\cos \left(z-\frac{\nu \pi}{2}-\frac{\pi}{4}\right)+\frac{4 \nu^{2}-1}{8 z} \sin \left(z-\frac{\nu \pi}{2}-\frac{\pi}{4}\right)+O\left(\frac{1}{z^{2}}\right)\right), \\
H_{\nu}^{(1)}(z) & =\sqrt{\frac{2}{\pi z}} e^{i\left(z-\frac{\nu \pi}{2}-\frac{\pi}{4}\right)}\left(1+i \frac{4 \nu^{2}-1}{8 z}+O\left(\frac{1}{z^{2}}\right)\right),
\end{aligned}
$$


here $O\left(1 / z^{2}\right)$ means that there exists a constant $C$ such that the remainder is bounded by $C / z^{2}$ for all $z \geq 1$. Taking the product and expanding $\cos \chi$ and $\sin \chi$ via the sum and difference of $e^{i \chi}$ and $e^{-i \chi}$, we obtain that

$$
J_{\nu}(z) H_{\nu}^{(1)}(z)=\frac{1}{\pi z}\left(1+e^{2 i\left(z-\frac{\nu \pi}{2}-\frac{\pi}{4}\right)}+i \frac{4 \nu^{2}-1}{4 z} e^{2 i\left(z-\frac{\nu \pi}{2}-\frac{\pi}{4}\right)}+O\left(\frac{1}{z^{2}}\right)\right)
$$

for all $z \geq 1$.

Remark A.1. One can easily deduce from (A.2), (A.3) and (A.10) that

$$
2 \rho \varphi_{\ell}^{0}(\rho, x) \psi_{\ell}^{0}(\rho, x)=i\left(1+e^{2 i\left(\rho x-\frac{(\ell+1) \pi}{2}\right)}+O\left(\frac{1}{\rho}\right)\right)
$$

and does not converge when $\rho \rightarrow \infty$ contrary to what is stated in [19, Remark 2.14].

We refer the reader to 28 , for the definition of the total variation of a function (denoted by $V(f ; \mathbb{R})$ ) and the class of bounded variation vanishing at infinity functions (denoted by $B V_{0}(\mathbb{R})$ ). We need the following two properties of the functions from $B V_{0}(\mathbb{R})$ class.

Lemma A.2. Let $f \in B V_{0}(\mathbb{R})$ and $g \in B V(\mathbb{R})$. Then $f \cdot g \in B V_{0}(\mathbb{R})$, and $V(f \cdot g ; \mathbb{R}) \leq V(f ; \mathbb{R})$. $\left(V(g ; \mathbb{R})+\|g\|_{L_{\infty}(\mathbb{R})}\right)$.

Proof. It is well-known that the product of two functions of bounded variation is again a function of bounded variation, see, e.g. [10], moreover

$$
V(f \cdot g ; \mathbb{R}) \leq V(g ; \mathbb{R}) \cdot \sup _{\mathbb{R}}|f|+V(f ; \mathbb{R}) \cdot \sup _{\mathbb{R}}|g| .
$$

Now the statement follows observing that for $B V_{0}$ functions one has $\sup _{\mathbb{R}}|f| \leq V(f ; \mathbb{R})$.

Lemma A.3 ([28, Corollary 10]). If $f \in B V_{0}(\mathbb{R})$, then for all $\omega \in \mathbb{R} \backslash\{0\}$ its Fourier transform $\hat{f}$ is defined and satisfies

$$
|\hat{f}(\omega)| \leq \frac{V(f ; \mathbb{R})}{|\omega|} .
$$

Now we can formulate the main result of this section.

Proposition A.4. Suppose that the potential $q \in L_{1}(0, \infty) \cap B V_{0}[0, \infty)$. Then the asymptotics (2.5) holds.

Proof. Due to the property $F_{\ell}(-\rho)=\overline{F_{\ell}}(\rho)$ we may assume that $\rho>0$. First we assume that $\ell>-1 / 2$. Let us rewrite (A.1) as

$$
\begin{aligned}
F_{\ell}(\rho)= & 1+\int_{0}^{1 / \rho} \psi_{\ell}^{0}(\rho, x) \varphi_{\ell}^{0}(\rho, x) q(x) d x+\int_{1 / \rho}^{\infty} \psi_{\ell}^{0}(\rho, x) \varphi_{\ell}^{0}(\rho, x) q(x) d x \\
& +\int_{0}^{\infty} \psi_{\ell}^{0}(\rho, x)\left(\varphi_{\ell}(\rho, x)-\varphi_{\ell}^{0}(\rho, x)\right) q(x) d x=: 1+I_{1}+I_{2}+I_{3} .
\end{aligned}
$$

The integral $I_{1}$ can be estimated using (A.4) and noting that functions of bounded variation are bounded,

$$
\left|I_{1}\right| \leq C^{2} \int_{0}^{1 / \rho} \frac{x|q(x)|}{1+\rho x} d x \leq \frac{C^{2}}{\rho} \int_{0}^{1 / \rho}|q(x)| d x \leq \frac{C_{1}}{\rho^{2}} .
$$


To estimate the integral $I_{2}$ we utilize (A.2), (A.3) and (A.10) and obtain

$$
\begin{aligned}
I_{2}= & \frac{i}{2 \rho} \int_{1 / \rho}^{\infty} q(x) d x+\frac{i e^{-i(\ell+1) \pi}}{2 \rho} \int_{1 / \rho}^{\infty} q(x) e^{2 i \rho x} d x \\
& -\frac{\ell(\ell+1) e^{-i(\ell+1) \pi}}{2 \rho^{2}} \int_{1 / \rho}^{\infty} \frac{q(x)}{x} e^{2 i \rho x} d x+\frac{i}{2 \rho} \int_{1 / \rho}^{\infty} q(x) O\left(\frac{1}{(\rho x)^{2}}\right) d x \\
= & I_{4}+I_{5}+I_{6}+I_{7} .
\end{aligned}
$$

Now we have

$$
I_{4}=\frac{i}{2 \rho} \int_{0}^{\infty} q(x) d x-\frac{i}{2 \rho} \int_{0}^{1 / \rho} q(x) d x=\frac{i}{2 \rho} \int_{0}^{\infty} q(x) d x+O\left(\frac{1}{\rho^{2}}\right)
$$

where we used that $q$ is bounded. To estimate the integral in $I_{5}$ note that it can be considered as $\int_{-\infty}^{\infty} g(x) e^{2 i \rho x} d x$, where $g(x)=\tilde{q}(x) \cdot \mathbf{1}_{[1 / \rho, \infty)}(x), \tilde{q}$ is an extension of $q$ to $\mathbb{R}$ by zero and $\mathbf{1}_{A}$ is the characteristic function of the set $A$. Both functions $\tilde{q}$ and $\mathbf{1}_{[1 / \rho, \infty)}$ are of bounded variation and one can easily see from Lemma A.2 that $V(g ; \mathbb{R}) \leq 4 V(q ;[0, \infty))$. Now applying Lemma A.3 we obtain that

$$
\left|I_{5}\right|=\frac{1}{2 \rho}\left|\int_{-\infty}^{\infty} g(x) e^{2 i \rho x} d x\right| \leq \frac{4 V(q ;[0, \infty))}{4 \rho^{2}} .
$$

The estimate for the integral $I_{6}$ is similar noting that the function $\frac{1}{x} \mathbf{1}_{[1 / \rho, \infty)}(x) \in B V_{0}(\mathbb{R})$ and $V\left(\frac{1}{x} \mathbf{1}_{[1 / \rho, \infty)}(x) ; \mathbb{R}\right) \leq 2 \rho$. Recalling the meaning of the $O$ symbol, we have for $I_{7}$

$$
\left|I_{7}\right| \leq \frac{C}{2 \rho^{3}} \int_{1 / \rho}^{\infty} \frac{|q(x)|}{x^{2}} d x \leq \frac{C}{2 \rho^{3}} \int_{1 / \rho}^{\infty} \frac{C_{2}}{x^{2}} d x=\frac{C C_{2}}{2 \rho^{2}} .
$$

Finally, for the integral $I_{3}$ we utilize (A.4) and (A.6) and obtain

$$
\left|I_{3}\right| \leq C^{2} \int_{0}^{\infty} \frac{x|q(x)|}{1+\rho x}\left(\exp \left(C \int_{0}^{x} \frac{y|q(y)|}{1+\rho y} d y\right)-1\right) d x
$$

Since $\int_{0}^{x} \frac{y|q(y)|}{1+|\rho| y} d y \leq \frac{1}{\rho} \int_{0}^{x}|q(x)| d x \leq \frac{1}{\rho}\|q\|_{L_{1}(0, \infty)}$, we have

$$
\left|\exp \left(C \int_{0}^{x} \frac{y|q(y)|}{1+\rho y} d y\right)-1\right| \leq \exp \left(\frac{C\|q\|_{L_{1}(0, \infty)}}{\rho}\right)-1=O\left(\frac{1}{\rho}\right)
$$

hence $I_{3}=O\left(\frac{1}{\rho^{2}}\right)$. Combining all the estimates we obtain the statement. Now assume that $\ell=-1 / 2$. From all the integrals $I_{1}, \ldots, I_{7}$ only the integrals $I_{1}$ and $I_{3}$ have to be treated differently from the case $\ell>-1 / 2$. For the first we use the estimate (A.5) and boundedness of $q$ and obtain

$$
\left|I_{1}\right| \leq C^{2} \int_{0}^{1 / \rho} \frac{x|q(x)|}{1+\rho x}\left(1-\log \frac{|\rho| x}{1+|\rho| x}\right) d x \leq \frac{C^{2} C_{q}}{\rho}\left(\frac{1}{\rho}-\int_{0}^{1 / \rho} \log \frac{|\rho| x}{1+|\rho| x} d x\right)=\frac{C_{3}}{\rho^{2}}(1+\log 4) .
$$

For the second integral note that since the function $q$ is bounded, the function $\bar{q} \in L_{1}(0, \infty)$, hence the same proof with the replacement of $q$ by $\bar{q}$ works. 


\section{References}

[1] M. Abramovitz and I. A. Stegun, Handbook of mathematical functions, New York: Dover, 1972.

[2] Z. S. Agranovich and V. A. Marchenko, The inverse problem of scattering theory, Gordon and Breach, 1963.

[3] R. G. Airapetyan, I. V. Puzynin and Eu. P. Zhidkov, Numerical method for solving the inverse problem of quantum scattering theory. In: B. Apagyi et al. (eds.), Inverse and Algebraic Quantum Scattering Theory, Springer-Verlag, Berlin, 1997, 88-97.

[4] N. I. Akhiezer and I. M. Glazman, Theory of linear operators in Hilbert space, Dover, New York, 1993.

[5] K. Chadan, P. C. Sabatier, Inverse problems in quantum scattering theory. Second edition, Springer-Verlag, New York, 1989.

[6] H. Chébli, A. Fitouhi and M. M. Hamza, Expansion in series of Bessel functions and transmutations for perturbed Bessel operators, J. Math. Anal. Appl. 181 (1994), 789-802.

[7] M. Coz and Ch. Coudray, The Riemann solution and the inverse quantum mechanical problem, J. Math. Phys. 17 (1976), no. 6, 888-893.

[8] B. B. Delgado, K. V. Khmelnytskaya, V. V. Kravchenko, The transmutation operator method for efficient solution of the inverse Sturm-Liouville problem on a half-line, Math. Meth. Appl. Sci. 42 (2019) 7359-7366.

[9] A. Erdélyi, Higher trascendental functions, vol. I, New-York-Toronto-London: McGraw-Hill, 1953.

[10] R. Gordon, W. College, Real analysis: a first course, 2nd edition, Pearson, 2002.

[11] R. L. Greene and C. Aldrich, Variational wave functions for a screenefl Coulomb potential, Phys. Rev. A 14 (1976), 2363-2366.

[12] M. Holzleitner, Transformation operators for spherical Schrödinger operators, J. Math. Anal. Appl. 481 (2020), 123430, 27 pp.

[13] M. Holzleitner, A. Kostenko and G. Teschl, Dispersion estimates for spherical Schrödinger equations with critical angular momentum, in: Partial Differential Equations, Mathematical Physics, and Stochastic Analysis, F. Gesztesy et al. (eds), EMS Congress Reports 14 (2018), 319-347.

[14] L. V. Kantorovich and G. P. Akilov, Functional analysis. Translated from the Russian by Howard L. Silcock. Second edition, Pergamon Press, Oxford-Elmsford, N.Y., 1982.

[15] A. N. Karapetyants, K. V. Khmelnytskaya and V. V. Kravchenko, A practical method for solving the inverse quantum scattering problem on a half line, J. Phys.: Conf. Ser. 1540 (2020), 012007, $7 \mathrm{pp}$.

[16] V. V. Katrakhov and S. M. Sitnik, The transmutation method and boundary value problems for singular elliptic equations, Sovrem. Mat. Fundam. Napravl. 64 (2018), issue 2, 211-426 (in Russian).

[17] A. Kostenko, A. Sakhnovich and G. Teschl, Inverse eigenvalue problems for perturbed spherical Schrödinger operators, Inverse Problems 26 (2010), 105013 (14pp).

[18] A. Kostenko and G. Teschl, Spectral asymptotics for perturbed spherical Schrödinger operators and applications to quantum scattering, Comm. Math. Phys. 322 (2013), no. 1, 255-275.

[19] A. Kostenko, G. Teschl and J. H. Toloza, Dispersion estimates for spherical Schrödinger equations, Ann. Henri Poincaré 17 (2016), 3147-3176. 
[20] V. V. Kravchenko, On a method for solving the inverse Sturm-Liouville problem, J. Inverse Illposed Probl. 27 (2019), 401-407.

[21] V. V. Kravchenko, On a method for solving the inverse scattering problem on the line, Math. Meth. Appl. Sci. 42 (2019), 1321-1327.

[22] V. V. Kravchenko, Direct and inverse Sturm-Liouville problems: A method of solution, Birkhäuser, Cham, 2020.

[23] V. V. Kravchenko, E. L. Shishkina and S. M. Torba, On a series representation for integral kernels of transmutation operators for perturbed Bessel equations, Math. Notes 104 (2018), 552-570.

[24] V. V. Kravchenko and S. M. Torba, Transmutation operators and a new representation for solutions of perturbed Bessel equations. Submitted. Available at arXiv:2005.10403v4.

[25] V. I. Kukulin and R. S. Mackintosh, The application of inversion to nuclear scattering, J. Phys. G: Nucl. Part. Phys. 30 (2004) R1-R55.

[26] B. M. Levitan, Inverse Sturm-Liouville problems, VSP, Zeist, 1987.

[27] S. T. Ma, On the Coulomb and Hulthén potentials, Aust. J. Phys. 7 (1954) 365-372.

[28] F. J. Mendoza-Torres, A note on two classical theorems of the Fourier transform for bounded variation functions, Communications in Mathematics and Applications, 7 (2016), No. 2, 73-80.

[29] S. G. Mihlin, The numerical performance of variational methods, Wolters-Noordhoff publishing, Groningen The Netherlands, 1971.

[30] R. Piessens, The Hankel transform. In: The Transforms and Applications Handbook: Second Edition, CRC Press, 2000.

[31] A. P. Prudnikov, Yu. A. Brychkov and O. I. Marichev, Integrals and series. Vol. 2. Special functions, New York: Gordon \& Breach Science Publishers, 1986, 750 pp.

[32] N. Setô, Bargmann's inequalities in spaces of arbitrary dimension, Publ. RIMS, Kyoto Univ. 9 (1974) 429-461.

[33] S. M. Sitnik, E. L. Shishkina, Method of transmutations for differential equations with Bessel operators, Fizmatlit, Moscow, 2019, 224 pp. (in Russian).

[34] E. L. Shishkina, S. M. Sitnik, Transmutations, singular and fractional differential equations with applications to mathematical physics, Elsevier, Amsterdam, 2020.

[35] A. S. Sohin, On a generalization of Levinson's theorem (in Russian), Teor. Funk., Funk. Analiz y Prilozheniya, 22 (1975) 136-145.

[36] V. V. Stashevskaya, The inverse problem of spectral analysis for differential operators with a singularity at the origin, Kharkov. Uchenye zapiski Kharkov. Mat. Obsch. (1957), no. 5, 49-86.

[37] G. Teschl, Mathematical methods in quantum mechanics. With applications to Schrödinger operators, 2nd ed., Amer. Math. Soc., Rhode Island, 2014.

[38] V. Ya. Volk, On inversion formulas for a differential equation with a singularuty at $x=0$, Uspehi Matem. Nauk (N.S.), 8 (56) (1953), no. 4, 141-151.

[39] S. Zaman and Siraj-ul-Islam, On numerical evaluation of integrals involving oscillatory Bessel and Hankel functions, Numer. Algorithms 82 (2019) 1325-1343. 